\title{
C/I BALANCING UNDER LIMITED POWER DYNAMICS FOR JD-CDMA
}

\author{
Rajanish, Student Member, IEEE and P.W. Baier, Fellow, IEEE
}

\begin{abstract}
In cellular mobile communications, to optimize spectrum efficiency of a cell net, carrierto-interference-ratio $(\mathrm{C} / \mathrm{I})$ balancing by the centralized power control (CPC) would be the optimum power control technique. CPC might be difficult to implement due to its heavy required signaling load. However, the study of CPC gives enlightening insight into the mechanism relevant to power control in mobile radio systems. CPC requires a large dynamic range of the transmission powers at the base stations (BSs), say of the order of $100 \mathrm{~dB}$ and sometimes more. This is practically not feasible, and hence there is a requirement for adequate power control techniques which work satisfactorily under the limited power constraint. The dynamic range of power typical for state-of-the-art transmitter amplifiers is very limited, for e.g., to a maximum of $20 \mathrm{~dB}$ as quoted by manufacturers and operators. In this paper, novel procedures for adjusting the transmitter powers under the consideration of limited dynamic range are envisaged to provide maximum possible $C / I$ ratio at the receivers. Obviously, the $C / I$ performance would degrade by a certain amount due to the limitation of the transmit power. The target system envisaged is the Time Divided - Code Division Multiple Access (TD-CDMA) system utilizing Joint Detection (JD). Monte Carlo simulations have been performed to evaluate the performance.
\end{abstract}

Keywords-C/I Balancing, Power Control, JDCDMA.

\section{INTRODUCTION}

The requirement of large spectrum efficiency has led to the development of efficient multiple access techniques like TD-CDMA utilizing Joint Detection (JD) [1]. JD has a distinct advantage of eliminating the intra-cell cochannel interference thus improving the spectrum efficiency. However, the spectruxn efficiency is governed by $C / I$ ratio at the mobile stations

Rajanish is with the Electrical Communication Engineering Department, Indian Institute of Science, Bangalore - 560012 , India.email: rajanishoprotocol.ece.iisc.ernet.in

P.W. Baier is with the Research Group of RF Communications, P.O.Box 3049, D-67653, University of Kaiserslautern, Germany.email: baierorhrk.uni-kl.de
(MSs). To maintain the optimum $C / I$ ratio, many power control procedures based on Aein [2] have been developed. The centralized power control (CPC) technique [3], [4] is a viable choice. But, CPC might be difficult to implement due to its heavy required signaling load. However, the study of CPC gives enlightening insight into the mechanism relevant to power control in mobile radio systems. To implement CPC, we need a large dynamic range of power at the B$\mathrm{S}$, say of the order of $100 \mathrm{~dB}$ and sometimes more, which is not available with the current state-of-theart amplifiers at higher power levels. This aspect has been completely ignored in the literature. In this paper, concepts and algorithms which would give. a new distribution of powers for the MSs, within the available dynamic range providing least degradation from the optimum $C / I$, have been proposed. The available dynamic range of the power (maximum to minimum ratio of the power) at the BSs, considering the state-of-the-art technology, is a maximum of $20 \mathrm{~dB}$, as per the manufacturers and operators. In $C / I$ balancing, it should be borne in mind, that the smaller the variance of the $C / I$, the better is the system. In this paper, chiefly, two algorithms are proposed, viz., the hard decision (HD) and the soft decision (SD) to obtain the $C / I$ ratio under the limited power dynamics. A comparison of the two is also brought out to choose the best algorithm. In order to obtain the $C / I$ performance closer to the $C / I$ performance under the unlimited power dynamics, research leading to the enhancement of the dynamic range of the power in the amplifiers is necessary. Hereafter, we would call the assumption of the unlimited power dynamics as the ideal condition. In this regard, it is necessary to obtain a fair idea of how much dynamic range would be sufficient to get approximately the same $C / I$ performance as obtained under the ideal condition. Owing to this, we have also investigated the effect of different dynamic power ranges like $30 \mathrm{~dB}, 40 \mathrm{~dB}$ and so on. Monte Carlo simulations are carried out to sirnulate the concept. The system performance is illustrated by plotting the cumulative distribution function (CDF) of the $C / I$ ratio in a cell net with the different random 
configurations of the MSs.

\section{Cellular System}

The cellular system simulated in this paper consists of regular hexagonal cells with no overlap. The BSs are placed in the center of each cell. The MSs are uniformly distributed with an average of $K$ MSs per cell. The BSs and the MSs employ omnidirectional antennas. BSs and MSs in the cell net are represented by the sets $\mathbb{B}=\left\{\beta_{1}, \beta_{2}, \ldots, \beta_{b}, \ldots, \beta_{\|\mathbb{E}\|}\right\}$ and $\mathbb{M}$ $=\left\{\mu_{1}, \mu_{2}, \ldots, \mu_{m}, \ldots, \mu_{\| \mathbb{M} \mid}\right\}$, respectively. Each MS $\mu_{\tilde{m}} \in \mathbb{M}$ is served by exactly one BS $\beta_{z(\tilde{m})} \in \mathbb{B}$, where the function $z(\tilde{m})$ establishes the relation between the MS $\mu_{\tilde{m}}$ and the BS $\beta_{b}$ to which this MS is assigned. The line of sight (LOS) link with a single direction of arrival (DOA) of the signal is considered at the BS, which is reasonable assumption for a macro-cellular system. Primarily, in a macrocellular system, the signals arrive at the BS through a smaller angular range around the LOS even if the LOS is shadowed [5]. The DOA valid for a certain uplink is also assumed to be valid for the corresponding downlink. The radio channel model considered for the system level simulation is based on the large-scale path loss component and the slowly varying component having a log-normal distribution. For each link between a BS and a MS, a certain propagation loss is assumed based on the above components. The large-scale path loss component is characterized by the distance $\rho_{z(m), \tilde{m}}$ between the BS $\beta_{z(m)}$ and the MS $\mu_{\dot{m}}$, and the attenuation coefficien$\mathrm{t} \alpha$. The slowly varying component is represented by a log-normal random variable $\xi_{z(m), \tilde{m}}$. The lognormal random variable, $\xi_{z(m), \tilde{m}}$, is derived form the Gaussian random variable $\chi$ with zero mean and a standard deviation $\sigma$ by a transformation given in [6]. The reciprocal of the propagation loss as the propagation power gain between the corresponding BS and the MS. The propagation gain between a certain BS $\beta_{z(m)}$ which serves $\mathrm{MS} \mu_{m}$ and a certain $\mathrm{MS} \mu_{\tilde{m}}$ is

$$
g_{z(m), \tilde{m}}=\frac{1}{\xi_{z(m), \tilde{m}} \cdot \rho_{z(m), \tilde{m}}^{\alpha}}
$$

The propagation gains $g_{z(\tilde{m}), m}$ and $g_{z(\tilde{\tilde{m}}), m}$ to a MS $\mu_{m}$ are equal if the BSs $\beta_{z(\tilde{m})}$ and $\beta_{z(\tilde{m})}$ are identical.

\section{C/I BALANCING}

$\mathbb{I}_{\rightarrow,}$ is the interference power received by the MS $\mu_{\tilde{m}}$ from the BSs $\beta_{z(m)}$ meant to serve the MSs $\mu_{m}$, $\mu_{m} \in \mathbb{M} \backslash \mu_{\hbar} . \mathbb{I}_{\hbar} \subset \mathbb{M}$ is the set of all the MSs $\mu_{m} \in \mathbb{M}$ causing interference to $\mathrm{MS} \mu_{\tilde{m}}$. The set of interferers $\mathbb{I}_{5}$ depends on the type of the detection scheme used in the system. Here, we have used TDCDMA using JD. The interfering signals are assumed to be statistically independent so that the interfering powers can be added to yield the total interfering power $I_{T_{\tilde{m}}}$. The carrier power for $\mu_{\tilde{m}}$ is denoted by $C_{\tilde{m}}$. With the transmission power $\mathcal{T}_{m}$, of a mobile $\mu_{m} \in \mathbb{M}$, the carrier-to-interference-ratio $\gamma_{\tilde{m}}$ for a link between the MS $\mu_{\tilde{m}}$ and the BS $\beta_{z(\tilde{m})}$ in the system is given by

$$
\gamma_{\tilde{m}}=\frac{C_{\tilde{m}}}{I_{T_{\tilde{m}}}}=\frac{T_{\tilde{m}} \cdot g_{z(\tilde{m}), \tilde{m}}}{\sum_{\left\{m \mid \mu_{m} \in \mathbb{I}_{\tilde{B}}\right\}}\left(T_{m} \cdot g_{z(m), \tilde{m}}\right)}
$$

$\gamma_{\bar{m}}$ depends on the transmission powers of all the mobiles corresponding to the propagation loss between the MS $\mu_{\tilde{m}}$ and the serving BS of each mobile. Hence $\gamma_{m}$ of different MSs $\mu_{m}$ are dependent on each other and cannot be adjusted independently. Thus, our goal should be to adjust the transmission powers for all the mobiles in such a way that, depending on all the propagation losses, the values $\gamma_{m} \forall \mu_{m} \in \mathbb{M}$ become equal and attain the maximum possible value $\Gamma$. This approach is termed $\mathrm{C} / \mathrm{I}$ balancing. It is showri in [3] that the $C / I$ balancing can be performed for a limited number of cells by solving an eigenvalue problem. In the case of $C / I$ balancing equation (2) can be written as

$$
\sum_{\left\{m \mid \mu_{m} \in \mathbb{I}_{\tilde{m}}\right\}} T_{m} \cdot g_{z(m), \tilde{m}}=\frac{1}{\gamma_{\tilde{m}}} \cdot T_{\tilde{m}} \cdot g_{z(\tilde{m}), \tilde{m}}
$$

The set of these equations for all || $\mathbb{M}||$ MSs gives a matrix vector equation. Denoting $\mathbf{W}$ as the matrix containing the propagation gains of the interferers, $g_{z(m), \tilde{m}}, \mu_{m} \in \mathbb{I}_{\dot{b}}, \mu_{\tilde{m}} \in\{\mathbb{M} \backslash \mathbb{I}\}, \mathbf{V}$ as the matrix containing the propagation gains of the carriers, $g_{z(\tilde{m}), \tilde{m}}$ and $t$ as a vector of all the transmission powers $T_{m}$, the matrix vector equation can be written as

$$
W \cdot t=\frac{1}{\Gamma} \cdot V \cdot t
$$

This eigenvalue problem contains the positive definite matrices $\mathbf{W}$ and $\mathbf{V}$. The solution of this equation is the balanced $C / I, \Gamma$, for which their exists all nonnegative $\|\mathbb{M}\|$ transmission powers $T_{m}, \mu_{m} \in \mathbb{M}$. The proof of this could be found in [3], [7].

\section{LIMITED POWER DYNAMICS}

The eigenvalue problem so obtained on the basis of the above discussions gives the transmission powers $T_{m}$ for $\mu_{m} \in \mathbb{M}$ which may take values in the range from 
zero to infinity. Clearly, such an infinitely extended power distribution is not feasible with real world transmitter amplifiers. The width of the dynamic power band available through state-of-the-art amplifiers amounts to a maximum of $20 \mathrm{~dB}$, as per the manufacturers and the operators. This leads to finding a new set of transmission powers, $\dot{T}_{m} \in\left(T_{\max }, T_{\min }\right)$, for each mobile $\mu_{m}$ under the same transmitting conditions, which would give the best possible $C / I$ at the $\mu_{m}$. In the logarithmic scale the limit primarily considered in the paper is given by

$$
\frac{T_{\max }}{T_{\min }}=20 \mathrm{~dB}
$$

where $T_{\max }$ and $T_{\min }$ are the maximum and minimum transmitted powers, i.e., $\max (t)$ and $\min (t)$, respectively. The improvement of the $C / I$ performance under the increased transmission power limit is also investigated which gives information to the hardware designers as to what dynamic range of power at the BSs would be required to obtain near to ideal performance.

Balancing $C / I$ under a limited power range of 20 $\mathrm{dB}$ leads to solving the eigen value problem (4) with a constraint on the upper and the lower power level which is computationally cumbersome. Hence, a simple procedure was developed to obtain a new set of transmission powers for the MSs from the old set of transmission powers obtained with an assumption of infinite power availability and then the corresponding $C / I$ of the cell net could be maximized. But, this poses a threat to the concept of $C / I$ balancing, because after forcing the limited power distribution, we obtain different $C / I$ at all the MSs in the cell net for one particular snapshot. This problem is circumvented by choosing the minimum $C / I$ of the snapshot to be the balanced $C / I$ in the cell net. Thus, it ensures that the system performance could be quite better than what is depicted in the results. In the first development towards this, a method of Hard Decision was developed which is explained below.

\section{A. Hard Decision}

The Hard Decision is, basically, restricting the transmission of powers for the MSs according to the availability at the BS, i.e., it is a technique which forces the BS to transmit less or more power, $\dot{T}_{m}$, for a particular mobile, $\mu_{m} \in \mathbb{M}$, depending on whether the power desired by the mobile, $T_{m}$, under ideal condition is larger or smaller than $T_{m a x}$ or $T_{m i n}$, respectively. It amounts to overlaying a $20 \mathrm{~dB}$ power band over the transmission power distribution obtained under the ideal condition and finding out the power band of width $20 \mathrm{~dB}$ which would give the maximum possible $C / I$ as compared to power bands of the same width. The algorithm followed to achieve this is as follows : For the transmission power $T_{m}>T_{\max }$

$$
\dot{T}_{m}=T_{\max }
$$

For the transmission power $T_{m}<T_{\text {min }}$

$$
T_{m}^{\prime}=T_{\min }
$$

For the transmission power $T_{\min }<T_{m}<T_{\max }$

$$
T_{m}^{\prime}=T_{m}
$$

This gives a new set of powers, $\dot{t}=\left\{T_{m}^{\prime}\right\}$, to be served to the mobiles, $\mu_{m} \in \mathbb{M}$, derived by imposing the power limit constraint. Now the power distribution is such that the mobiles having $T_{m}>T_{\max }$ get lesser power than $T_{m}$, thus reducing the interference, the mobiles having $T_{m}<T_{\min }$ get more power than the desired $T_{m}$, thus, increasing the interference and the mobiles having powers $T_{\min }<T_{m}<T_{\max }$ get the same power $T_{m}$ as desired under the unlimited power dynamics. Now, our aim is to calculate the $C / I$ for each MS $\mu_{m}$ with the new power distribution, $T_{m}^{\prime}$. The new set of $C / I, \dot{\gamma}_{\tilde{m}}$, for all the MS $\mu_{m} \in \mathbb{M}$ is calculated by replacing the new powers in (2)

$$
\dot{\gamma}_{\tilde{m}}=\frac{C_{\tilde{m}}}{I_{\dot{T}_{\tilde{m}}}}=\frac{\dot{T}_{\tilde{m}} \cdot g_{z(\tilde{m}), \tilde{m}}}{\sum_{\left\{m \mid \mu_{m} \in \mathbb{I}_{\tilde{\tilde{m}}}\right\}}\left(\dot{T}_{m} \cdot g_{z(m), \tilde{m}}\right)}
$$

Solving the above equation for each MS gives different $C / I$ unlike the unlimited power dynamics. To obtain the new CDF of the $C / I$ in this case, we choose the minimum of $\dot{\gamma}_{\tilde{m}}$ of all the MSs, $\mu_{m} \in \mathbb{M}$, which is the worst $C / I$ to be achieved in order to serve all the MSs. Thus, balancing the cell net at this $C / I$ would provide better system performance than depicted in the results.

Now to obtain the best possible $C / I$ for $20 \mathrm{~dB}$ power band a search algorithm is invented. First, a power band of $20 \mathrm{~dB}$ is selected by selecting the lower bound of the power band, $T_{l}$, equal to the minimum power required by a mobile in the case of unlimited power dynamics and the upper bound, $T_{u}$ equal to $T_{l}+20 \mathrm{~dB}$. By employing this algorithm, new $C / I$, $\dot{\gamma}$, is calculated as per (6) for all the mobiles $\mu_{m} \in \mathbb{M}$ The minimum of $\hat{\gamma}_{\tilde{m}}$ is considered to be the $C / I$ which should be implemented in order to serve all the mobiles in the cell net in that particular instant. Then, a new power band is selected by taking the lower bound 
of power band, $T_{l}$, equal to the next minimum power required by a mobile in an unlimited power dynamics and the upper bound, $T_{u}$, equal to $T_{l}+20 \mathrm{~dB}$. The minimum of $\dot{\gamma}_{\tilde{m}}$ is calculated again. This search process is repeated $\|\mathbb{M}\|$ times, equal to the number of MSs. Out of $\|\mathbb{M}\|$ power bands, the best power band is the one which attempts the maximum of the minimum $\dot{\gamma}_{\tilde{m}}$ calculated. We can observe here that the number of search attempts is directly proportional to the number of MSs. Thus, to reduce the number of search, it requires a knowledge of some parameter that can approximately predict the mean of the best power band. We have also observed in our simulations that the mean of the best power band lies close to the mean of the powers obtained under the ideal condition. This is discussed in detail with the result$s$ in the subsequent section containing the simulation results.

\section{B. Soft Decision}

It is noted above that making a hard decision forces the transmitted power to a mobile to be lower or higher than the desired, $T_{m}$, (under unlimited power dynamics) causing deterioration in the system performance which could be observed in the results presented in the subsequent section. Moreover, the search algorithm needed to find out the best power distribution becomes computationally inefficient as the number of MSs increases in a cell net, of course discarding the rough estimates for finding the best power band. This led to the development of the algorithms which would recalculate the new set of powers to be transmitted under the limited power constraint, reducing or eliminating the search process for the best power band. This we term the soft decision (SD) process. In this paper, two different algorithms are presented in order to make $\mathrm{SD}$. The new set of transmitted powers, $T_{m}^{\prime}$, are calculated either by compressing the adjacent differences between the powers to be transmitted to the MSs or by compressing the adjacent ratios between the powers to be transmitted. The use of this algorithm eliminates the search process for the best power band. This is explained in detail in the following subsections.

B.1 Method of Compression of the Adjacent Difference (M

In this algorithm the new set of powers, $T_{m}^{\prime}$, to be transmitted to the MSs are obtained by the following algorithm :

- For a given set of transmitted powers, $t=\left\{T_{m}^{\prime}\right\}$, for MSs, $\mu_{m} \in \mathbb{M}$, under the unlimited power dynamics, arrange them in the ascending/descending order.

- Calculate the difference between the adjacent powers as

$$
d_{i}=T_{i+1}-T_{i} \quad i=1, \ldots . .,(m-1)
$$

where $\mathrm{m}=$ number of MSs

- Compute the compression unit, $C_{d}$

$$
C_{d}=\frac{T_{a_{\max }}-T_{a_{\min }}}{T_{\max }-T_{\min }} \quad i=1, \ldots .,(m-1)
$$

where $T_{a_{m a x}}$ and $T_{a_{\min }}$ are the maximum and minimum bound of the power band available for the transmission.

- Modify the adjacent difference, $d_{i}$ to $D_{i}$

$$
D_{i}=d_{i} * C_{d} \quad i=1, \ldots,(m-1)
$$

- Obtain the new set of powers, $T_{m}$

$$
\begin{gathered}
\dot{T}_{1}=T_{r_{\text {min }}} \\
\dot{T}_{i}=T_{i-1}^{\prime}+D_{i-1} \quad i=2, \ldots ., m
\end{gathered}
$$

The new set of the transmitted powers, $t=\left\{T_{m}\right\}$, so obtained are substituted in (6) to calculate the new $C / I, \dot{\gamma}$. But, unlike the unlimited power case, here we would obtain || $\mathbb{M} \|$ different $C / I$, too, like it was using the HD. Hence, to obtain the CDF of the $C / I$ in this case, too, we choose the minimum of $\dot{\gamma}_{\bar{m}}$ of all the MSs $\mu_{m} \in \mathbb{M}$ which is the worst $C / I$ to be achieved in order to serve all the MSs.

It was also discovered that the new set of transmission powers, $\boldsymbol{t}$, could also be obtained by another technique involving compression of the adjacent ratios of the transmitted powers, $t$, obtained under the ideal condition. The use of this algorithm may require a search for finding the exponents for finding the best compression unit, but the search time is very small as compared to the $\mathrm{HD}$, as it is independent of the number of MSs unlike HD. The algorithm to do so functions as follows

B.2 Method of Compression of the Adjacent Ratio (MCAR,

- For a given set of transmitted powers, $t$, for MSs IOAD $\in \mathbb{M}$ under the unlimited power dynamics, arrange them in ascending/descending order.

- Calculate the ratio between the adjacent powers as

$$
r_{i}=T_{i+1} / T_{i} \quad i=1, \ldots,(m-1)
$$

where $\mathrm{m}=$ number of MSs 


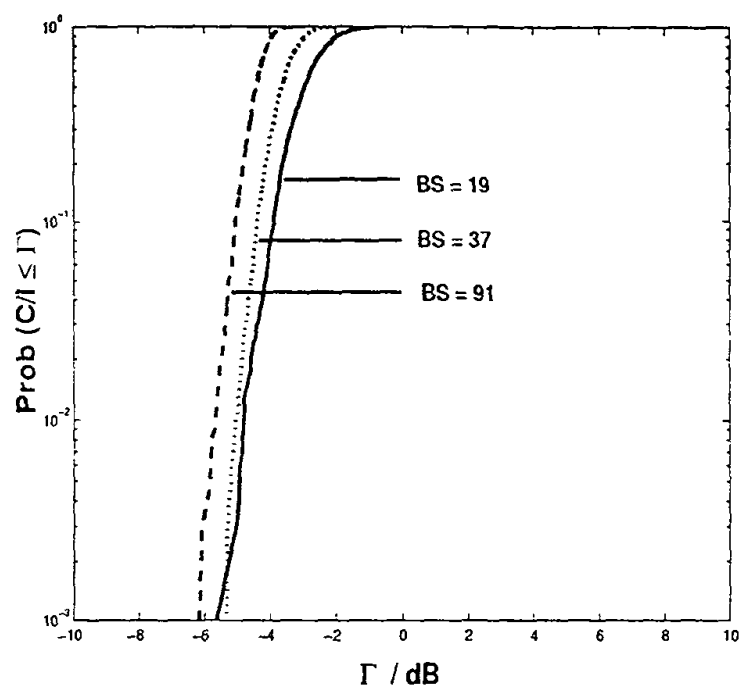

Fig. 1. CDF of $C / I$ for the various number of cells/BSs with an average of $4 \mathrm{MSs}$ per BS

- Compute the compression unit, $C_{r_{i}}$

$$
C_{r_{i}}=\frac{\left(T_{a_{\max }} / T_{a_{\min }}\right)^{x_{i}}}{T_{\max } / T_{\min }} \quad i=1, \ldots,(m-1)
$$

where, $x_{i}$ is the $i^{\text {th }}$ element of the arithmetic progression (AP) series whose sum is 1 , where, $x \geq 0$. Geometric progression (GP) could also be used to determine the series $x_{i}$, but it is computationally inefficient and hence discarded. The AP series is obtained as

$$
x_{i}=\frac{2 \cdot\{1+(i-1) \cdot b\}}{i \cdot\{2+(i-1) \cdot b\}}
$$

where, $b$ is the progression difference which could be set to a value that would give the best power distribution for obtaining the best $C / I$.

- Modify the adjacent ratio, $r_{i}$, to $R_{i}$

$$
R_{i}=r_{i} \cdot C_{\tau_{i}} \quad i=1, \ldots,(m-1)
$$

- Obtain the new set of powers, $\dot{t}=\left\{T_{m}^{\prime}\right\}$, as

$$
\begin{gathered}
\dot{T}_{1}=T_{a_{\text {min }}} \\
\dot{T}_{i}=T_{i-1}^{\prime} \cdot D_{i-1} \quad i=2, \ldots ., m
\end{gathered}
$$

\section{Simulation Results}

To study the system performance with the limited power dynamics the CDF of $C / I$ of the cell net, $\Gamma$, is investigated. It is desired that the CDF curve of $\Gamma$ should be as steep as possible because this confirms the smaller variance of $\Gamma$. The cellular environment

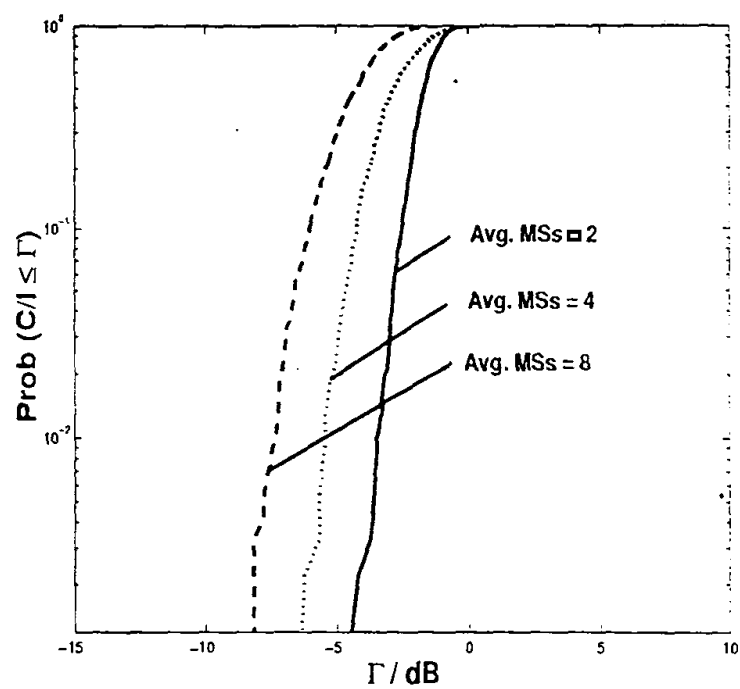

Fig. 2. CDF of $C / I$ for the various average density of MSs per BS

considered consists of regular hexagonal cells arranged in tiers with BSs located at the center of each cell. The MSs are uniformly distributed over the complete cell net. The antenna is omnidirectional at all the BSs and the MSs. Each MS is served by the BS to which it has got the highest propagation gain. It is also assumed that each BS can serve an unlimited number of MSs, which reflects that call blocking is ignored. The interference is exclusively intercell because of the JD scheme assumed which eliminates intracell interference. The other parameters are $\sigma=8 \mathrm{~dB}, \alpha=4$ and reuse factor of one. Owing to the limited simulation capacity, the cell net considered for the simulation consists of 2, 3 and 5 tiers of the cells, i.e., 19, 37 and $91 \mathrm{BSs}$, respectively, with a uniform distribution of an average number of 2 to $8 \mathrm{MSs}$ per BS. The results are obtained as CDF of $C / I$ for one thousand snapshots of MSs distributions. Throughout the paper, the system performance is compared for the outage probability of $1 \%$.

To study the effect of the size of the cell net on the $C / I$ performance, simulations were carried out using 19, 37 and 91 BSs with an average of four MSs per BS. Figure 1 depicts the comparison of the CDF of $C / I$ which reflects the degradation of the system performance with the increase in the systern size. This degradation is expected because of the increase in the number of interferers for each MS.

Figure 2 shows the CDF of $C / I$ for the differen$t$ average density of MSs per BS in a cell net of 37 BSs. We can observe that for every doubling of the 


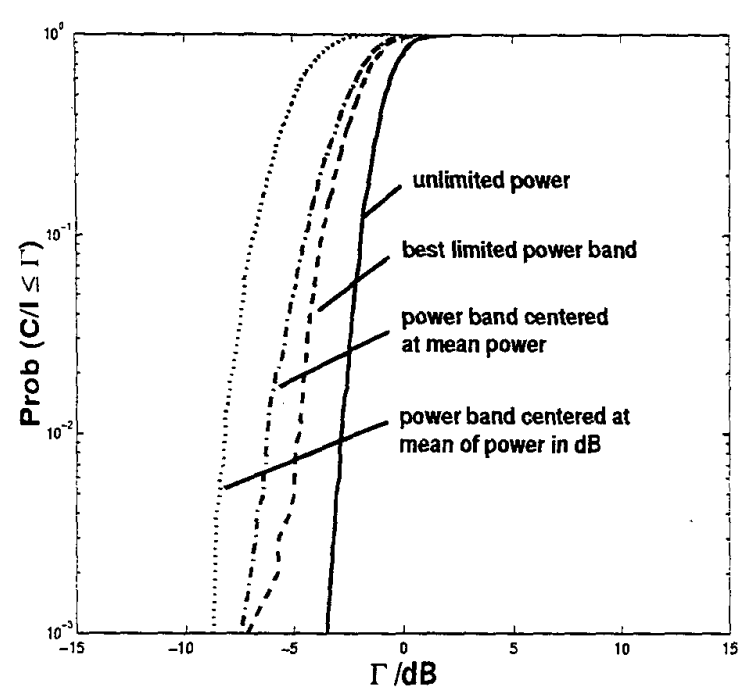

Fig. 3. CDF of $C / I$ with the unlimited and limited power of $20 \mathrm{~dB}$ using the $\mathrm{HD}$, depicting the effect of different choice of power bands for $37 \mathrm{BSs}$ and an average of 2 MSs per BS

average MSs, there is $2 \mathrm{~dB}$ degradation in the overall $C / I$ of the cell net. This also could be attributed to the increase in the cumulative interference. It could also be pointed out that, if we increase the number of cells with nearly uniform density of the MSs, then the degradation of overall $C / I$ is not very severe as compared to the $C / I$ deterioration in the case of increasing the density of the MSs with the number of cells, i.e., BSs kept fixed because of the interferer diversity.

The $\mathrm{HD}$ as mentioned above, would give a best possible power band that would maximize the possible $C / I$ in the cell net. However, expense of the search process is proportional to the number $\|\mathbb{M}\|$ of MSs in the cell net. In a bid to do reduce the search, we obtained the $C / I$ performance in the cell net with three different power bands. The first power band is obtained by the regular search with the search steps equal to the number $\|\mathbb{M}\|$ of MSs, the second power band is obtained when the mean of the required power band is equal to the mean of the transmission power distribution obtained under the ideal condition, and the third power band is obtained when the mean of the required power band is equal to the the mean of the transmission powers in $\mathrm{dB}$ under the ideal condition. The performance of the system obtained by these procedures is shown in Figure 3. We can observe that the best performance is obtained if the power band is selected for the maximum $C / I$ through the proposed search process. But, more importantly, it is observed

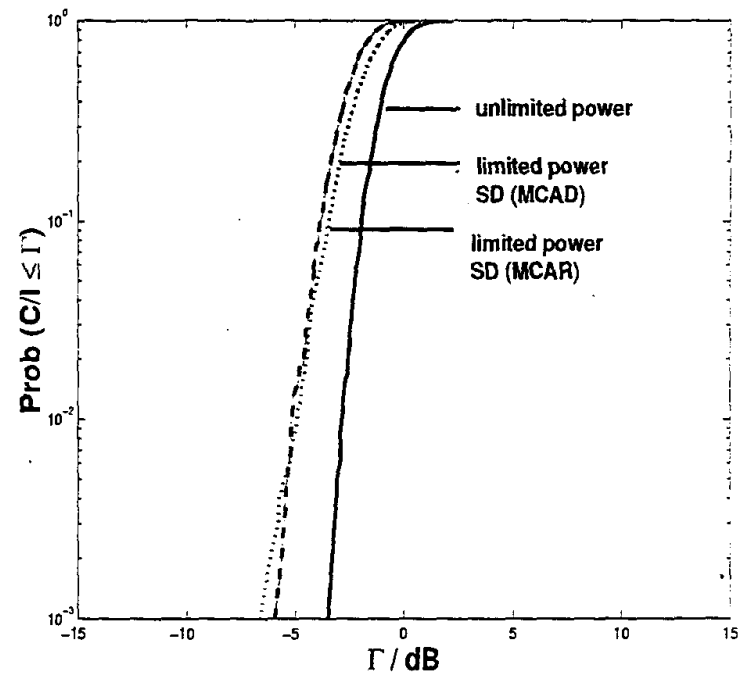

Fig. 4. CDF of $C / I$ showing the comparison of the algorithms for SD for $37 \mathrm{BSs}$ and an average of $2 \mathrm{MSs}$ per BS

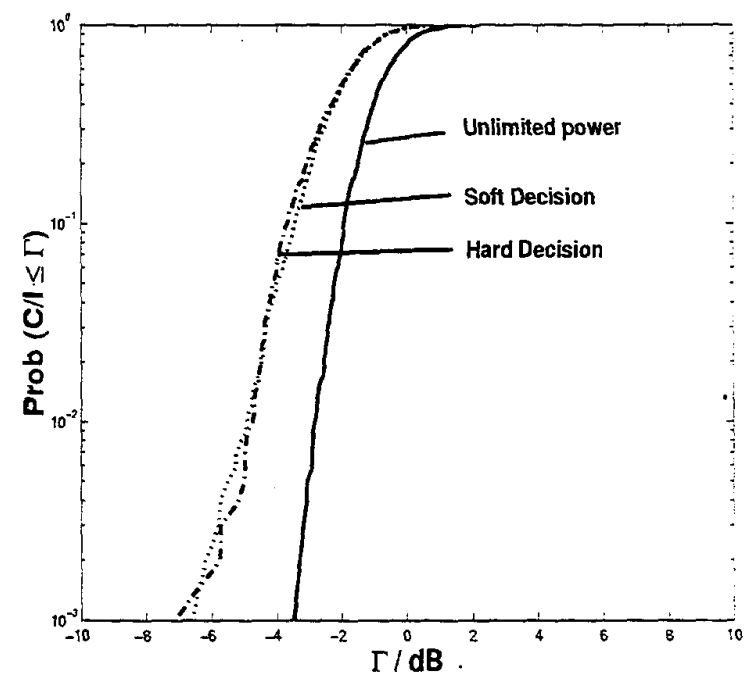

Fig. 5. CDF of $C / I$ to compare the HD and the SD for 37 BSs and an average of 2 MSs per BS

that the $C / I$ performance obtained from the power band whose mean is centered at the mean of the transmission powers is closer to the performance obtained using the regular method.

In order to reduce/eliminate the search process, a novel algorithm of SD was developed for which two procedures were envisaged, namely MCAD and MCAR discussed above in detail. The performance under both these cases are compared in Figure 4. We see that MCAD gives a slightly better performance than MCAR. Moreover, the computation time for the M- 


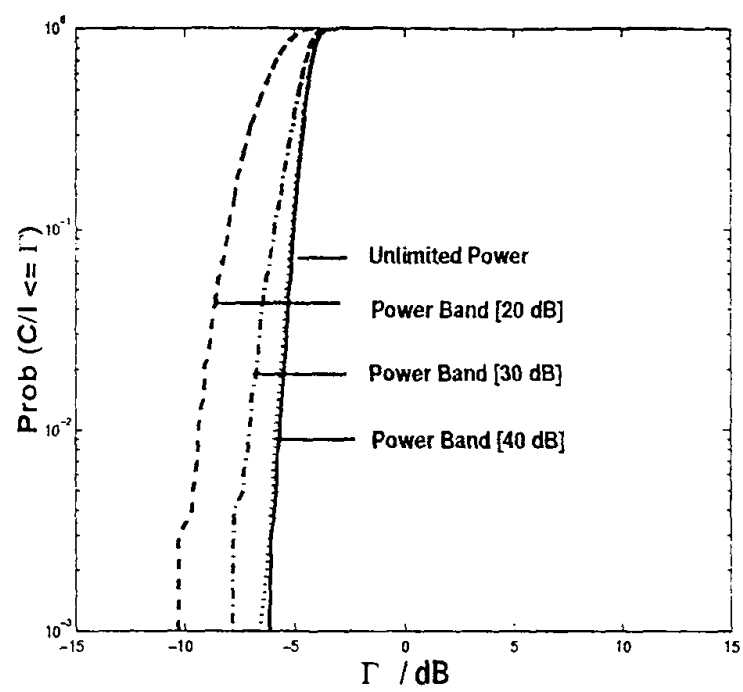

Fig. 6. CDF of $C / I$ with the different limited power bands for the distribution of 4 MSs on an average for 91 BSs

CAD is less than that for MCAR. Thus, either of the two procedures of SD would be computationally less expensive than the $\mathrm{HD}$, but given one choice, MCAD would be the best. The SD and the HD are then compared in Figure 5 which tells us that the hard decision made by choosing the best power band is equally good compared to the soft decision making, but SD would be computationally less expensive as compared to $\mathrm{HD}$.

As a matter of interest and for the knowledge of the hardware designers of the amplifiers, the limit of the dynamic power was increased to explore the maximum dynamic range of the power needed at the BSs in the centralized power control strategy for obtaining the closest $C / I$ performance to the one obtained under the assumption of the unlimited power control. The $C / I$ performance was studied for $20 \mathrm{~dB}, 30 \mathrm{~dB}$, and $40 \mathrm{~dB}$ and is presented in Figure 6 . It can be observed from the results that the performance under 40 $\mathrm{dB}$ is nearly equal to the performance obatined under for ideal condition for even relatively large scenario of 91 BSs with an average of 4 MSs per BS. Thus, $40 \mathrm{~dB}$ availability would be sufficient for the cellular mobile communications employing JD-CDMA for the best QoS, with an optimum usage of the resources.

\section{Conclusions}

Two novel methods, viz., Hard Decision and Soft Decision have been presented in this paper to obtain $C / I$ balancing under the limited power dynamics. It could be concluded that the soft decision using the method of the compression of the adjacent difference could be employed to obtain the best performanice under the limited power dynamics. It is computationally less expensive as compared to the hard decision algorithm. But, HD with the estimation strategy based on the mean of the transmitted powers obtained under infinite power assumption also gives comparable performance. It is also concluded that the availability of the dynamic range of $40 \mathrm{~dB}$ at the BSs would be sufficient to get nearly the ideal performance for the best QoS with an optimum usage of the resources.

\section{ACKNOWLEDGMENTS}

The authors would like to thank Mr. Ralph Schmalenberger, Mr. Yang Lu and the other colleagues at Research Group of RF Cornmunications for the useful exchange of ideas. The authors would also like to express their sincere thanks to Prof. M. Pandit, DAAD( Deutscher Akademischer Austauschdienst) and Prof. T. S. Vedavathy, Indian Institute of Science, Bangalore, India, for providing the opportunity for this work. The authors would also like to express sincere thanks to the University of Kaiserslautern, Germany and the Indian Institute of Science, Bangalore, India for providing the platform for this work.

\section{REFERENCES}

[1] P. Jung and J. Blanz, "Joint detection with coherent receiver antenna diversity in CDMA mobile radio systems with joint detection", IEEE Trans. on Vehicular Tech., Vol. 44, pp. $76-88,1995$.

[2] J. M. Aein, "Power balancing in systems employing frequency reuses," COMSAT Tech. Rev., vol. 3, no.2, Fall 1973.

[3] Grandhi,S.A.,Vijayan, R., Goodman, D.J.,Zander, J., "Centralized power control in cellular radio systems",IEEE Trans. on Veh. Tech., vol. 42, no.4, pp. 466-468, Nov 1993.

[4] Schmalenberger R., Blanz J. J., "Multi antenna $C / I$ balancing in the downlink of digital cellular mobile radio systems", Proc. IEEE 47th Vehicular Technology Conference, VTC'97(Phoenix), pp 607-611.

[5] Klein, A., Mobr, W., Thomas, R., Weber, P., Wirtb, B., "Direction- of-arrival of partial waves in wideband mobile radio channels for intelligent antenna concepts", Proc. IEEE 46th Vehicular Technology Conference VTC'96, pp 849-853.

[6] Steil, A., "Statistics of the carrier-to-interference ratio in C/TDMA cellular mobile radio systems applying multiuser detection", Wireless Personal Communications - An International Journal (Kluwer), 1997.

[7] Minc, H.,"Nonnegative Matrices", John Wiley and Sons, new York, 1988. 